\title{
Progress in Epithelial-Mesenchymal Transition Research
}

\author{
Donald F. Newgreen $^{\mathrm{a}}$ Erik W. Thompson ${ }^{\mathrm{b}, \mathrm{c}}$ \\ aEmbryology Laboratory, Murdoch Children's Research Institute, Royal Children's Hospital, Parkville, Vic., \\ bUniversity of Melbourne Department of Surgery, St. Vincent's Hospital, and 'Invasion and Metastasis Unit, \\ St. Vincent's Institute, Fitzroy, Vic., Australia
}

The field of research of epithelial-mesenchymal transitions, EMT, and its reverse, mesenchymal-epithelial transitions, MET, has expanded very rapidly indeed from its beginnings, heralded by Professor Betty Hay in the 1970s and 1980s. This expansion has involved the realisation that the EMT was not just an interesting phenomenon of early developmental morphogenetic cell behaviour, but bore remarkable resemblance to clinically crucial pathological events in cancer invasion. Not surprisingly, this discipline soon became numerically dominant in the EMT publication field. Simultaneously, the EMT concept has been extended to normal physiological wound healing. Exploration revealed that these resemblances were more than skin deep: the same sets of growth factors, receptors, transcription factors, epigenetic marks and signalling pathways turned up repeatedly in EMTs and METs in a variety of contexts, both pathological and normal. This molecular genetic research in turn uncovered similarities of the EMT signature to that of fibrosis, a set of diseases which is of enormous clinical importance, rivalling that of cancer. Most recently, and more surprisingly, the EMT signature has shown considerable similarity to that found in stem cell and cancer stem cell biology.

The explosive growth of the EMT field and the parallel progress of the TEMTIA and other EMT meetings were summarised in the last of our previous Special Issues with
Cells Tissues Organs [Thompson et al., 2011]. The TEMTIA meetings were inaugurated in 2003 explicitly to bridge across all these disciplines, so that experts in the various fields - who rarely attended the same conferences - could mutually benefit. It is fair to say that these biannual meetings have been enormously successful in their aims and influential across their fields. In the TEMTIA-V meeting held in Singapore, October 10-13, 2011, a vast amount of new information was presented and this clearly demonstrated the power provided by the new genetic and quantitative recording technologies when applied to EMT.

Articles in this special topic series: 1. Said, N.A.B.M., K.J. Simpson, E.D. Williams (2013) Strategies and challenges for systematically mapping biologically significant molecular pathways regulating carcinoma epithelial-mesenchymal transition. Cells Tissues Organs 197: 424-434. 2. Vargas, D.A., O. Bates, M.H. Zaman (2013) Computational model to probe cellular mechanics during epithelial-mesenchymal transition. Cells Tissues Organs 197: 435-444. 3. Elsum, I.A., P.O. Humbert (2013) Localization, not important in all tumor-suppressing properties: a lesson learnt from Scribble. Cells Tissues Organs 198: 1-11. 4. Kulesa, P.M., J.A. Morrison, C.M. Bailey (2013) The neural crest and cancer: a developmental spin on melanoma. Cells Tissues Organs 198: 12-21.

\section{KARGER}

E-Mail karger@karger.com

www.karger.com/cto
(C) 2013 S. Karger AG, Basel

$1422-6405 / 13 / 1976-0421 \$ 38.00 / 0$
Prof. Erik Thompson

St. Vincent's Institute and University of Melbourne Department of Surgery Room 210, Clinical Sciences Building, St. Vincent's Hospital 29 Regent Street, Fitzroy, Melbourne, VIC 3065 (Australia) E-Mail rik@svi.edu.au 
These emerging technologies bring a danger that data acquisition at such massive levels will outstrip acquisition of understanding, and may even obfuscate instead of illuminate. This 'data overload' is a real problem, which brings to the fore the necessity of exploiting these technologies in a systematic and efficient manner. This is explored in a review by Said et al. [2013] in the context of carcinoma EMT.

The EMT phenomenon is complex at the genetic level, the subcellular biochemical and biomechanical level, and the cellular behavioural and biophysical level. It may be that even if biological molecular and cellular events are individually relatively simple, difficulties will arise when there are vast numbers of events occurring simultaneously and in series, and these events influence each other. The time-honoured approach of biological intuition strikes real difficulty in navigating through such spatiotemporally complex webs of interactions. Formal mathematical modelling can be of enormous help here, because multiple variables can be coded for and followed, with complex parallel/series interactions simulated. Of course, it is easy to say that mathematical modelling requires that the rules which define the formal model be appropriate for the biological archetype they are attempting to describe, but this is not so easy to achieve given that mathematicians and biologists sometimes seem to talk in different and mutually incomprehensible languages. Still, in recent years much progress has been made in mathematical formulations of biological phenomena. Here, Vargas et al. [2013] present a model exploring the relationship of the biophysics of the extracellular matrix to the motility of cells as they leave an epithelial sheet and intrude into matrix.

Much work on changes in cell behaviour such as the EMT has centred on the gain, loss or mutational modification of molecules in the cell. More recently, attention has been placed on a somewhat more subtle change, the functional effects of systematically altering the position (mislocalising) of a molecule in the cell. Using the polarity complex protein Scribble (originally discovered in a Drosophila developmental screen) in a breast cancer model, Elsum and Humbert [2013] point to the complexity of outcomes from mislocalisation, with certain functions disrupted but other functions maintained. More precise understanding of the molecular implications of mislocations in such epithelial regulators/mediators will be crucial to our informed interpretation of drug discovery screens that employ these endpoints.
One of the strengths of the developmental models of EMT is that developmental processes are under stringent control - they always occur in the same way. This facilitates the typical mode of biological experimentation, which is to disrupt some process and see what changes as a result. Thus, detailed knowledge of molecular, cell and tissue events like EMT has been built up over decades of research. For all their fine-scale genetic, molecular and cell behavioural similarities to developmental EMTs, cancer EMTs are aberrant functions and always have elements of unpredictability and variability. Partially to bring these under some systematic control, much research on cancer cells has been translated into in vitro models. This means that the investigator has control over the environment of the cancer cells, and thereby limits a major source of variation. Of course, this presupposes that lessons learnt in the dish can be translated into understanding in tissues, which is not always the case. Kulesa et al. [2013] merge the strengths of two major model EMT systems, one a major invasive cancer system, melanoma, and the other its very welldescribed developmental lineage precursor, the embryonic neural crest EMT and cell migration (invasion) system. This combination is used to explore cancer cell/ microenvironment interactions in vivo, in three-dimensional tissues. In doing so, they also exploit the new technologies and analytic techniques to overcome a major difficulty in embryo research, that of extremely small sample sizes.

Collectively, these articles speak to the heart of the TEMTIA-V meeting in facilitating key concepts and strategies that cross the various disciplines touched by EMT. Of course, many more subject areas were represented, with sessions on cell/molecular biology of EMT, developmental EMT, mathematical modelling of EMT and invasion, organ fibrosis, stem cells and EMT, cancer and EMT, and drug discovery and EMT. The meeting was convened by Jean Paul Thiery and Erik Thompson, with an international program committee comprising Bob Weinberg (USA), Raghu Kalluri (USA), Angela Nieto (Spain), Shoukat Dedhar (Canada), David Epstein (USA), Margaret Frame (UK), Don Newgreen (Australia), Pierre Savagner (France), Guojun Sheng (Japan), Alice Wong (Hong Kong), Hongquan Zhang (China) and Raymond Runyan (USA). The meeting attracted 224 registrants, including 48 students, 126 postdocs and 26 speakers. The esteemed Betty Hay Award for a junior postdoctoral scientist in this field was ultimately awarded to Sarah Dunlap for her outstanding work on regulation of EMT status and cancer progres- 
sion by obesity in mouse models. The Singapore meeting honoured the outstanding work of Dr. Angela Nieto with the Betty Hay Oration. It is timely that, as this special series of EMT papers goes to press, registration opens for the next TEMTIA-VI meeting in Alicante,
Spain, convened by current TEMTIA President Angela Nieto and Amparo Cano (www.emtmeeting.org). The 2015 meeting is set for Melbourne, Victoria, Australia, in October 2015, and will be convened by Erik Thompson and Don Newgreen.
References

Progress in EMT Research
Elsum, I.A., P.O. Humbert (2013) Localization, not important in all tumor-suppressing properties: a lesson learnt from scribble. Cells Tissues Organs 198: 1-11.

Kulesa, P.M., J.A. Morrison, C.M. Bailey (2013) The neural crest and cancer: a developmental spin on melanoma. Cells Tissues Organs 198: $12-21$.

Said, N.A.B.M., K.J. Simpson, E.D. Williams (2013) Strategies and challenges for systematically mapping biologically significant molecular pathways regulating carcinoma epithelial-mesenchymal transition. Cells Tissues Organs 197: 424-434.
Thompson, E. W., R. Runyan, P. Savagner, D.F. Newgreen (2011) Out of the desert: the 4th TEMTIA meeting on new advances in development, fibrosis and cancer. Cells Tissues Organs 193: 4-7.

Vargas, D.A., O. Bates, M.H. Zaman (2013) Computational model to probe cellular mechanics during epithelial-mesenchymal transition. Cells Tissues Organs 197: 435-444. 